\title{
An Optimization Approach For Noncoplanar Intensity-Modulated Arc Therapy Trajectories
}

\author{
Humberto Rocha ${ }^{1,2}$ [0000-0002-5981-4469], Joana Dias ${ }^{1,2}$ [0000-0003-2517-7905], \\ Tiago Ventura ${ }^{2,3}[0000-0003-1562-0387]$, \\ Brígida Ferreira ${ }^{2,4}[0000-0001-7988-7545]$, and Maria do Carmo \\ Lopes $^{2,3[0000-0001-7339-7342]}$ \\ 1 CeBER and Faculdade de Economia, Universidade de Coimbra, \\ 3004-512 Coimbra, Portugal \\ 2 INESC-Coimbra, \\ 3030-290 Coimbra, Portugal \\ 3 Serviço de Física Médica, IPOC-FG, EPE, \\ 3000-075 Coimbra, Portugal \\ 4 Escola Superior de Saúde, Politécnico do Porto \\ 4200-072 Porto, Portugal \\ hrocha@mat.uc.pt, joana@fe.uc.pt, tiagoventura@ipocoimbra.min-saude.pt, \\ bcf@ess.ipp.pt, mclopes@ipocoimbra.min-saude.pt
}

\begin{abstract}
The latest generation of linear accelerators allows the simultaneous motion of gantry and couch leading to highly noncoplanar arc trajectories. The use of noncoplanar trajectories in arc radiotherapy was recently proposed to combine the benefits of arc treatment plans, such as short treatment times, with the benefits of step-and-shoot noncoplanar intensity-modulated radiation therapy (IMRT) treatment plans, such as improved organ sparing. In this paper, a two-step approach for the optimization of highly noncoplanar arc trajectories is presented and tested using a complex nasopharyngeal tumor case already treated at the Portuguese Institute of Oncology of Coimbra. In the first step, a set of noncoplanar beam directions is calculated resorting to one of the beam angle optimization (BAO) algorithms proposed in our previous works for step-and-shoot IMRT. In the second step, anchored in the points (beam directions) calculated in the first step, the proposed optimization strategy determines iteratively more anchor points that will define the noncoplanar arc trajectory, considering the dosimetric criteria used for the noncoplanar BAO search rather than geometric or time criteria commonly used. For the patient tested, the resulting noncoplanar arc therapy plan has undoubtedly greater overall quality compared to both the coplanar arc therapy plan and the typically used coplanar equispaced step-and-shoot IMRT plan.
\end{abstract}

Keywords: noncoplanar radiotherapy, arc therapy, optimization, treatment planning 


\section{Introduction}

In classic step-and-shoot IMRT, a linear accelerator mounted on a gantry rotates around the patient delivering non-uniform radiation fields from a set of fixed coplanar beams. Allowing the rotation of the couch where the patient is laid results in noncoplanar beam irradiation which may improve treatment plan quality, particularly for complex intra-cranial tumors [1]. In arc therapy, irradiation is done continuously while the gantry rotates around the patient with the treatment beam always on. Nowadays, volumetric modulated arc therapy (VMAT) $[2,3,4]$ is considered one of the most efficient IMRT arc techniques, in particular regarding dose delivery time. VMAT treatment plans typically use coplanar beam trajectories, performed for a fixed couch angle (usually $0^{\circ}$ ), where irradiation of the patient is modulated by a multileaf collimator (MLC), determining the gantry speed.

The use of noncoplanar trajectories in VMAT was recently proposed to combine the benefits of arc therapy treatment plans, such as short treatment times, with the benefits of noncoplanar IMRT treatment plans, such as improved organ sparing. Yang et al. [5] used principal component analysis to optimize the collimator angle dynamically and considered a hierarchical clustering algorithm to calculate noncoplanar arc trajectories. MacDonald and Thomas [6] embedded beams-eye-view (BEV) dose metrics in their arc trajectory optimization. Smyth et al. considered a fluence based local search algorithm [7] and also embedded geometrical metrics in their arc trajectory optimization [8]. Papp et al. [9] and Wild et al. [10] considered the travel salesman problem to find the best arc trajectories between fixed angles. A similar approach aiming to improve computational time and plan quality was recently proposed by Langhans et al. [11].

In this paper, we propose a two-step approach for optimizing noncoplanar arc trajectories. In the first step, a set of noncoplanar beam directions is calculated resorting to one of the $\mathrm{BAO}$ algorithms proposed in our previous works for step-and-shoot IMRT [12]. In the second step, anchored in the points (beam directions) calculated in the first step, the proposed optimization strategy determines iteratively more anchor points that will define the noncoplanar arc trajectory, considering the same dosimetric criteria used for the noncoplanar $\mathrm{BAO}$ rather than geometric or time criteria commonly used. A nasopharyngeal tumor case, corresponding to a complex intra-cranial tumor treated at the Portuguese Institute of Oncology of Coimbra (IPOC), is used to illustrate and test the proposed optimization strategy. The remainder of the paper is organized as follows. The nasopharyngeal tumor case is described in the next Section. In Section three we present the strategy proposed for noncoplanar arc trajectory optimization. Computational results are presented in Section four followed by the conclusions and future work in the last Section.

\section{Nasopharyngeal Tumor Case}

A clinical nasopharyngeal tumor case treated at IPOC was used to illustrate and test our approach. Nasopharyngeal tumors are intra-cranial tumor cases 
Table 1. Prescribed doses for the planning target volumes and tolerance doses for the organs considered.

\begin{tabular}{lccc}
\hline Structure & \multicolumn{2}{c}{ Tolerance Dose } & Prescribed \\
\cline { 2 - 3 } & Mean & Max & dose \\
\hline PTV $_{\text {70 }}$ & - & - & $70.0 \mathrm{~Gy}$ \\
PTV 59.4 $_{\text {Spinal cord }}$ & - & - & $59.4 \mathrm{~Gy}$ \\
Brainstem & - & $45 \mathrm{~Gy}$ & - \\
Left parotid & $26 \mathrm{~Gy}$ & - & - \\
Right parotid & $26 \mathrm{~Gy}$ & - & - \\
Oral cavity & $45 \mathrm{~Gy}$ & - & - \\
Body & - & $80 \mathrm{~Gy}$ & - \\
\hline
\end{tabular}

requiring treatments that are particularly difficult to plan given the large number of organs that surround the tumor. The brainstem, the spinal cord, the parotids (the larger salivary glands) and the oral cavity (that contains the remaining salivary glands) are the organs-at-risk (OARs) considered. For safety purposes, the tumor volume is enlarged by adding a margin originating a structure called planning target volume (PTV). Two levels of radiation dose are prescribed: a higher radiation dose of $70 \mathrm{~Gy}$ is prescribed to the tumor (called $P T V_{70}$ ) and a lower radiation dose of $59.4 \mathrm{~Gy}$ is prescribed to the lymph nodes (called $P T V_{59.4}$ ).

Prescribed doses for the planning target volumes and tolerance doses for the organs considered are displayed in Table 1 . The spinal cord and the brainstem are serial organs. Serial organs are compromised even if only a small part is damaged and therefore maximum-dose constraints are considered for such type of organs. On the other hand, parallel organs functioning is not much affected if a small part of the organ is damaged and thus mean-dose objectives are considered for such type of organs. The oral cavity and the parotids are parallel organs. A structure, called Body, that corresponds to the remaining organs and tissues is also considered to prevent the deposition of high radiation doses elsewhere.

\section{Highly Noncoplanar Arc Trajectory Optimization}

The latest generation of linear accelerators allows the simultaneous motion of gantry and couch. A treatment plan that uses simultaneous gantry and couch rotation while the treatment beam is on will be considered, leading to a highly noncoplanar arc trajectory. In this study, we provide an optimization approach for the noncoplanar arc trajectory of a VMAT plan, called $4 \pi V M A T$, and compare it with the coplanar arc trajectory of a VMAT plan, called $2 \pi V M A T$, and with the typically used equispaced step-and-shoot IMRT plan, called Equi. Previous works on noncoplanar VMAT start by calculating a noncoplanar trajectory of the incident beam and then a fully VMAT plan is optimized along the calcu- 
lated trajectory. The main goal of this study is to present our optimization strategy for the noncoplanar arc trajectory. Instead of obtaining fully VMAT plans, the different plans will be compared considering unsequenced IMRT treatment plans.

The two-step approach that we propose for optimizing noncoplanar arc trajectories combines two optimization problems, the BAO problem and the arc trajectory optimization, that are quite challenging just by themselves. Furthermore, as a dosimetric criteria is used to guide these two optimization problems, the fluence map optimization (FMO) problem needs to be addressed as well. Formulation and resolution approaches used to address FMO and BAO problems are succinctly presented in the next two sub-sections, followed by the description of the proposed strategy for noncoplanar arc trajectory optimization.

\subsection{Fluence Map Optimization}

There are many different formulations for the FMO problem, some of them assuring an automated optimization procedure [13]. Some of the most used formulations consider a weighted sum of objectives and most of the times constraints are implemented as objectives as well. The objectives defined for target and organs are naturally conflicting, and it is difficult to define what is the best trade-off between them. Therefore, a multicriteria formulation for the FMO problem is the most appropriate formulation. A multicriteria approach based on a wish-list defined a priori is considered as proposed by Breedveld et al. [14,15,16].

The wish-list constructed for the nasopharyngeal tumor case in study is displayed in Table 2. Due to the complexity of the clinical case, it was necessary to computationally define a set of additional structures other than PTVs and OARs. $P T V_{59.4}$ shell is obtained by removing a $10 \mathrm{~mm}$ margin of $P T V_{70}$ to $P T V_{59.4}$ and its purpose is to prevent high doses in the lymph nodes. Ring $P T V_{59.4}$ and Ring $P T V_{70}$ are obtained by creating ring structures with $10 \mathrm{~mm}$ of thickness at $10 \mathrm{~mm}$ distance from $P T V_{59.4}$ and $P T V_{70}$, respectively. These two auxiliary structures aim at improving target coverage and conformity. Finally, External Ring is obtained by creating a ring of $10 \mathrm{~mm}$ thickness next to the patient outer contour. This auxiliary structure is used to prevent possible high entrance doses.

Following the prescribed and tolerance doses displayed in Table 1, a wishlist containing 9 hard constraints and 10 prioritized objectives was constructed. Constraints must be strictly fulfilled as all are maximum-dose type constraints. Following the order in the wish-list, defined a priori, objectives are sequentially optimized. For tumors, the logarithmic tumor control probability ( $L T C P)$ was considered for dose optimization [16],

$$
L T C P=\frac{1}{N_{T}} \sum_{l=1}^{N_{T}} e^{-\alpha\left(D_{i}-T_{i}\right)},
$$

where $T_{i}$ is the dose prescribed to the tumor, $D_{i}$ is the dose in voxel $i, N_{T}$ is the number of PTV voxels, and $\alpha$ is a parameter assessing cell sensitivity. Doses 
Table 2. Wish-list constructed for the nasopharyngeal tumor case.

\begin{tabular}{|c|c|c|c|c|c|c|}
\hline & Structure & Type & \multicolumn{4}{|c|}{ Limit } \\
\hline \multirow{10}{*}{ Constraints } & $P T V_{70}$ & maximum & \multirow{9}{*}{\multicolumn{4}{|c|}{$\begin{array}{c}74.9 \mathrm{~Gy}(=107 \% \text { of prescribed dose }) \\
63.6 \mathrm{~Gy}(=107 \% \text { of prescribed dose }) \\
63.6 \mathrm{~Gy}(=107 \% \text { of prescribed dose }) \\
45 \mathrm{~Gy} \\
54 \mathrm{~Gy} \\
59.5 \mathrm{~Gy}(=85 \% \text { of prescribed dose }) \\
50.5 \mathrm{~Gy}(=85 \% \text { of prescribed dose }) \\
45 \mathrm{~Gy} \\
70 \mathrm{~Gy}\end{array}$}} \\
\hline & $P T V_{59.4}$ & maximum & & & & \\
\hline & $P T V_{59.4}$ shell & maximum & & & & \\
\hline & Spinal cord & maximum & & & & \\
\hline & Brainstem & maximum & & & & \\
\hline & Ring $P T V_{70}$ & maximum & & & & \\
\hline & Ring $P T V_{59.4}$ & maximum & & & & \\
\hline & External Ring & maximum & & & & \\
\hline & Body & maximum & & & & \\
\hline & Structure & Type & Priority & Goal & Parameters & Sufficient \\
\hline \multirow{10}{*}{ Objectives } & $P T V_{70}$ & LTCP & 2 & 1 & $T_{i}=70 \mathrm{~Gy} ; \alpha=0.75$ & 0.5 \\
\hline & $P T V_{59.4}$ & LTCP & 1 & 1 & $T_{i}=59.4 \mathrm{~Gy} ; \alpha=0.75$ & 0.5 \\
\hline & $P T V_{59.4}$ shell & LTCP & 3 & 1 & $T_{i}=59.4 \mathrm{~Gy} ; \alpha=0.75$ & 0.5 \\
\hline & External ring & maximum & 4 & $42.75 \mathrm{~Gy}$ & - & - \\
\hline & Spinal cord & maximum & 5 & 42.75 Gy & - & - \\
\hline & Brainstem & maximum & 6 & $51.3 \mathrm{~Gy}$ & - & - \\
\hline & Parotids & mean & 7 & 50 Gy & - & - \\
\hline & Oral cavity & mean & 8 & $45 \mathrm{~Gy}$ & - & - \\
\hline & Parotids & mean & 9 & $26 \mathrm{~Gy}$ & - & - \\
\hline & Oral cavity & mean & 10 & $35 \mathrm{~Gy}$ & - & - \\
\hline
\end{tabular}

that are lower than the prescribed ones are penalized by $L T C P$, whilst doses $D_{i}$ higher than the prescribed ones will make $L T C P$ tend to zero. The objective is to obtain an $L T C P$ of one, which corresponds to a homogeneous dose equal to the prescribed dose $T_{i}$.

This formulation of the FMO problem is addressed by $2 p \epsilon c$, a primal-dual interior-point algorithm tailored for multicriteria IMRT treatment planning [14]. For more details on $2 p \epsilon c$ interior-point algorithm see Breedveld et al. [14].

\subsection{Noncoplanar Beam Angle Optimization}

The BAO problem is most of the times formulated as a combinatorial optimization problem, considering a discrete sample of all continuous beam angle directions. Computational time constraints make exhaustive search-like approaches prohibitive. A number of different approaches have been proposed to reduce the computational time, including simulated annealing [17], neighborhood search [18], gradient search [19], branch-and-prune [20], genetic algorithms [21], or hybrid approaches [22]. None of these approaches is able to calculate, in a polynomial run time, the optimal solution of the combinatorial BAO problem (NP hard problem) [23]. An alternative formulation of the BAO problem has been considered in our works. All possible continuous beam angle directions around the tumor have been considered instead of a discretized set of beam directions, leading to a continuous global optimization problem [24,25,26,27,28]. The continuous formulation of the noncoplanar BAO problem is briefly described next.

Let $n$ be defined a priori as the number of noncoplanar beam irradiation directions and denote the couch angle as $\phi$ and the gantry angle as $\theta$. As gantry 


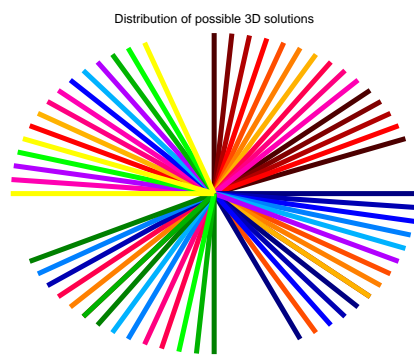

(a)

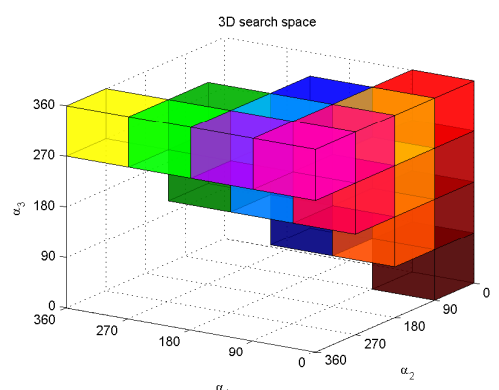

(b)

Fig. 1. All possible sorted combinations of three-beam angle sets divided by the four quadrants -1 (a) and the corresponding painted cubes in the reduced BAO search space $[0,360]^{3}-1(b)$.

angles $370^{\circ}$ and $10^{\circ}$ are the same irradiation directions (for a fixed couch angle), we consider an unbounded formulation. The continuous formulation of the noncoplanar BAO problem considers an objective function for which the best beam irradiation set corresponds to the function's minimum:

$$
\begin{aligned}
& \min f\left(\left(\theta_{1}, \phi_{1}\right), \ldots,\left(\theta_{n}, \phi_{n}\right)\right) \\
& \text { s.t. }\left(\theta_{1}, \ldots, \theta_{n}, \phi_{1}, \ldots, \phi_{n}\right) \in \mathbb{R}^{2 n} .
\end{aligned}
$$

The optimal value of the FMO problem has been used by us as the objective function, $f$, that guides the BAO search. In a noncoplanar beam irradiation setting, not all possible combinations of couch and gantry angles are feasible due to possible collision between the patient and the gantry. In order to maintain an unbounded formulation, noncoplanar irradiation directions that would cause collisions are penalized in the objective function as follows:

$$
f\left(\left(\theta_{1}, \phi_{1}\right), \ldots,\left(\theta_{n}, \phi_{n}\right)\right)= \begin{cases}+\infty & \text { if collisions occur } \\ \text { optimal FMO value } & \text { otherwise }\end{cases}
$$

The continuous BAO search space has a symmetric property explained by the simple fact that the order of irradiation directions is irrelevant in terms of optimization. By sorting the irradiation directions for each beam angle set, a large reduction of the BAO search space is obtained [12]. In previous works, we propose a multistart strategy that takes advantage of the symmetric feature of the continuous BAO search space. For an efficient sampling of the reduced BAO search space, the strategy sketched consists in considering all possible sorted combinations of beam angle sets divided by the four quadrants, as illustrated in Fig. 1(a) for a continuous three-dimensional BAO search space. Each of the represented three-beam angle set will define a starting point located in the different painted cubes illustrated in Fig. 1(b). 
The two main aspects of the proposed multistart approach, after the definition of the initial starting points, are the local procedure considered to explore each of the cubes of the reduced search space, and the definition of each cube as a region of attraction to avoid overlapping of different local search procedures. For the local search procedure, pattern search methods (PSM) as described in Rocha et al. [29] are used for their ability to avoid local entrapment. Algorithms 1 and 2 display the PSM algorithm and the multistart algorithm, respectively. For further details on the parallel implementation of the multistart approach see Rocha et al. [29].

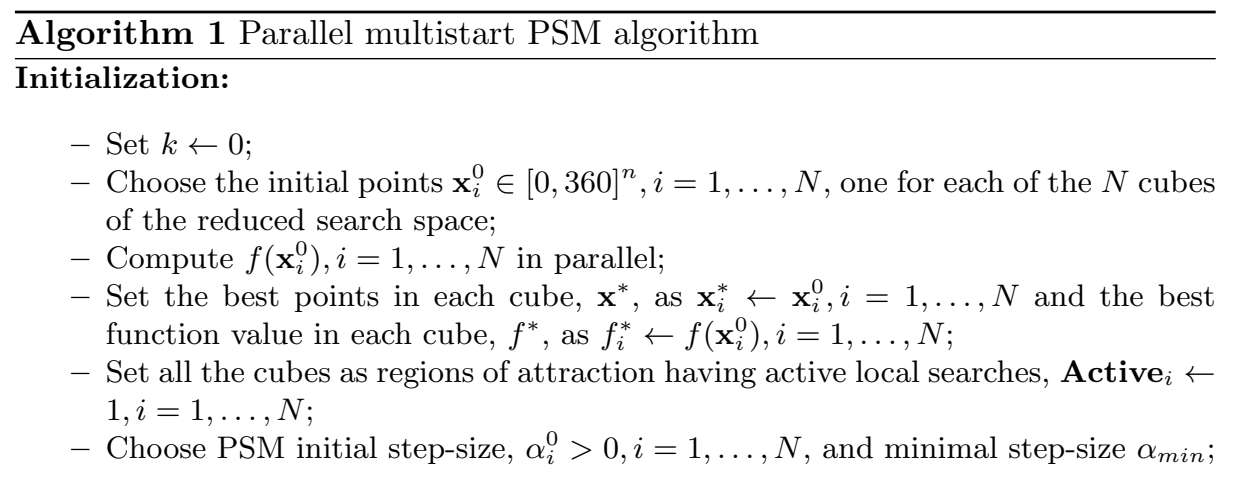

\section{Iteration:}

1. Use PSM (algorithm 2) to locally explore the cubes with active local search;

2. For cubes $i$ with active local search do

If $f\left(\mathbf{x}_{i}^{k}\right)<f\left(\mathbf{x}_{i}^{*}\right)$ then

If $\mathbf{x}_{i}^{k}$ is in cube $i$ then $\mathbf{x}_{i}^{*} \leftarrow \mathbf{x}_{i}^{k}$ $f_{i}^{*} \leftarrow f\left(\mathbf{x}_{i}^{k}\right)$

Else

Active $_{i} \leftarrow 0$

Determine cube $j \neq i$ where $\mathbf{x}_{i}^{k}$ is;

If $f\left(\mathbf{x}_{i}^{k}\right)<f\left(\mathbf{x}_{j}^{*}\right)$ then

$\mathbf{x}_{j}^{*} \leftarrow \mathbf{x}_{i}^{k}$;

$f_{j}^{*} \leftarrow f\left(\mathbf{x}_{i}^{k}\right)$

Active $_{j} \leftarrow 1$

Else

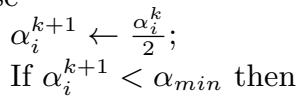

3. If there exists active cubes go to first step and set $k \leftarrow k+1$. 


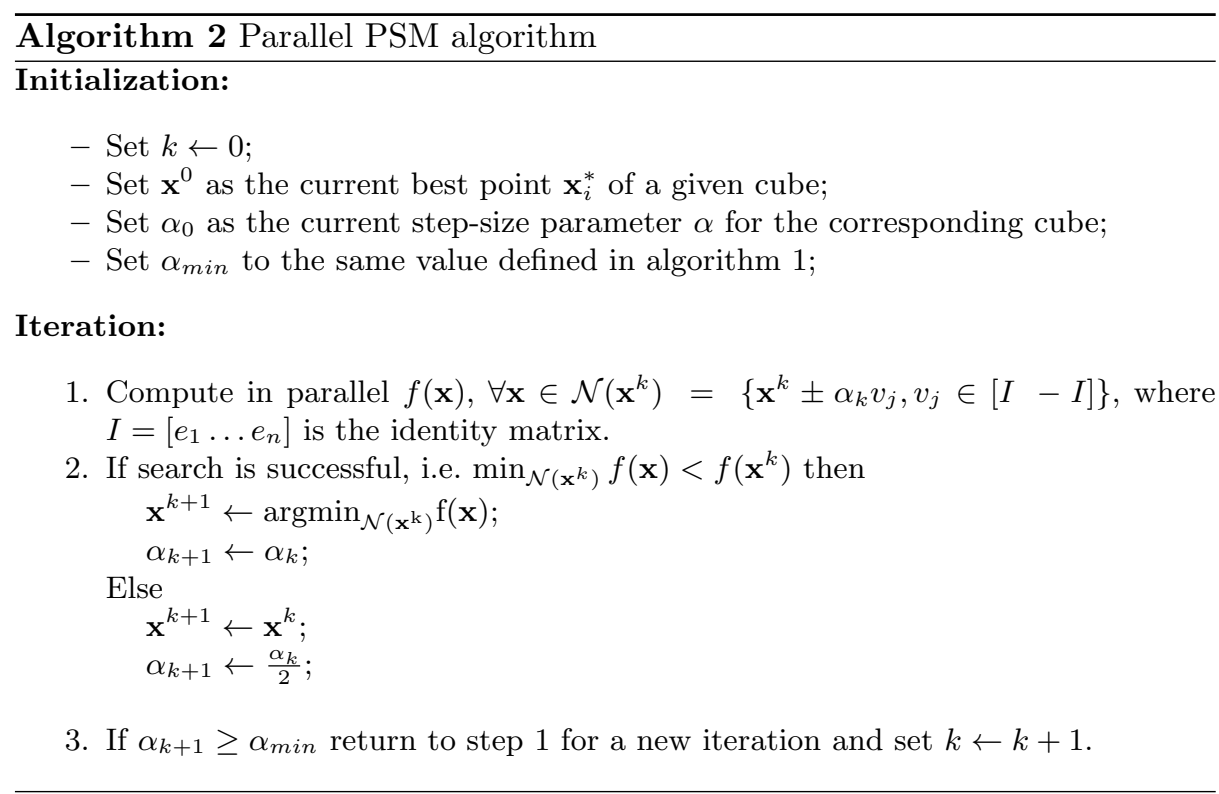

\subsection{Noncoplanar Arc Trajectory Optimization}

The optimization approach proposed for calculating noncoplanar arc trajectories is divided into two steps. In the first step, using the BAO algorithm described in Section 3.2, a set of noncoplanar beam directions is calculated. Then, in the second step, anchored in the points obtained by the BAO algorithm, novel anchor points are added iteratively considering only optimal FMO values. This iterative procedure ends when 20 anchor points are obtained, which is the typical number of anchor points considered in the literature (see, e.g., $[9,11])$. This second step is now described considering the nasopharyngeal tumor case to illustrate the optimization strategy sketched.

Although the BAO optimization procedure described in Section 3.2 explores the search space in a continuous manner, by defining appropriately the step-size parameter, $\alpha$, we end up with integer solutions. If the initial step-size parameter, $\alpha_{0}$, is a power of 2 , and the initial point is a vector of integers, by halving the step-size parameter in unsuccessful iterations we obtain integer iterates until the step-size parameter becomes inferior to 1 . This possibility is rather interesting for the BAO problem and was adopted in our PSM implementation.

For this second step, instead of considering the fine discretization of the BAO search space $\left(1^{\circ}\right)$, resulting from the outcome of integer solutions, we consider an equispaced beam grid separated by $10^{\circ}$ for both the gantry and the couch. Note that finer beam grids separated by $5^{\circ}, 2^{\circ}$ or $1^{\circ}$ can be considered at a cost of larger computational times. After exclusion of infeasible couch-gantry angle pairs due to possible collisions of patient and gantry for a nasopharyngeal tumor case, we end up with 472 candidate beams homogeneously distributed as 


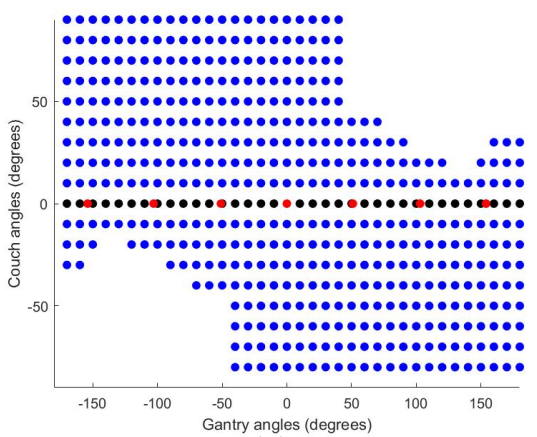

(a)

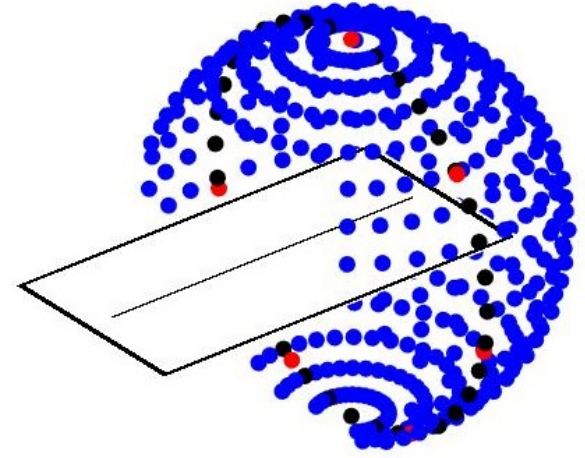

(b)

Fig. 2. Candidate beams homogeneously distributed represented in $2 \mathrm{D}-2$ (a) and the corresponding 3D representation - 2(b). Black beams correspond to the coplanar (couch fixed at $0^{\circ}$ ) $2 \pi$ VMAT while red beams correspond to the 7-beam coplanar equispaced solution, commonly used in clinical practice for step-and-shot IMRT.

illustrated in Fig. 2. The coplanar (couch fixed at $0^{\circ}$ ) $2 \pi$ VMAT and the 7-beam coplanar equispaced solution commonly used in clinical practice for step-andshoot IMRT, are also displayed in Fig. 2, corresponding to the black and red points, respectively.

The initial anchor points corresponding to the 7-beam noncoplanar BAO solution for the nasopharyngeal tumor case at hand are displayed in red in Fig. 3. For simplicity, the anchor points displayed correspond to the closest points in the equispaced beam grid. Some of the criteria commonly used for calculating noncoplanar arc trajectories include geometrical and time considerations. Our approach, similarly to the $\mathrm{BAO}$ approach, is based on dosimetric considerations, and will be guided by the optimal values of the FMO problem. Nevertheless, aiming to enhance one of the main features of VMAT, short treatment times, the following constraints are considered for the movement of the gantry/couch:

- The initial gantry/couch position is the beam of the 7-beam noncoplanar BAO solution with lower gantry angle value, corresponding to the leftmost anchor point in Fig. 3(a);

- The next anchor point to visit is the anchor point with lower gantry angle value among the ones that have not yet been visited;

- The final gantry/couch position is the beam of the 7-beam noncoplanar $\mathrm{BAO}$ solution with higher gantry angle value, corresponding to the rightmost anchor point in Fig. 3(a);

- When moving from one anchor point to the next one, the gantry must move towards the next anchor point while the couch must move towards the next anchor point or be halted.

The main goal of these movement restrictions is to define a trajectory from the leftmost anchor point to the rightmost anchor point of Fig. 3(a) as fast as 


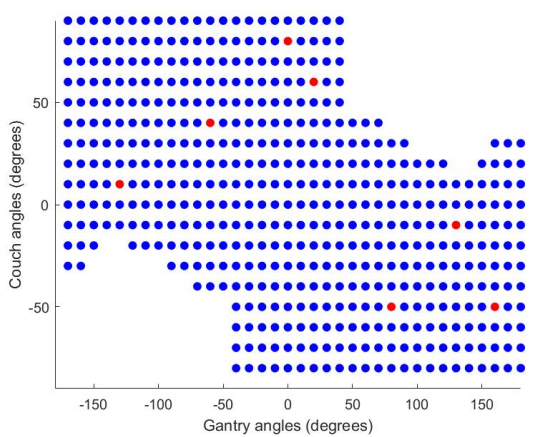

(a)

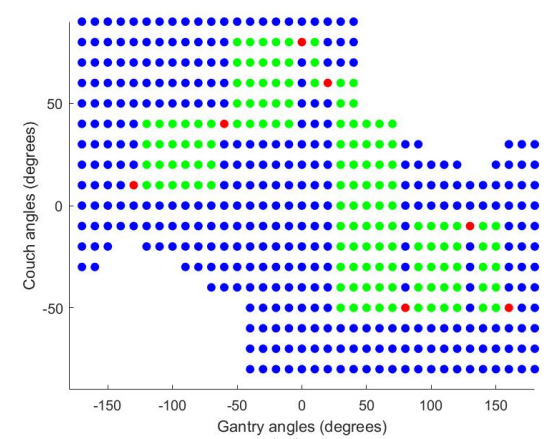

(b)

Fig. 3. The 7-beam noncoplanar BAO solution is displayed in red - 3(a) and the feasible points to consider when calculating a new anchor point are displayed in green $-3(b)$.

possible, i. e., with the gantry always rotating towards the next anchor point and the couch always moving (when this is the case) towards the next anchor point. Defining in this way the possible gantry and coach movements, the feasible points to consider when calculating a new anchor point are shown in green in Fig. 3(b).

There are different ways of considering the optimal value of the FMO problem to iteratively add novel anchor points, one by one. The most expensive, in terms of computational time, is to add each one of the green points, one at a time, to the existing set of anchor points and then compute the corresponding optimal FMO value considering these beams. The candidate beam that leads to the minimum optimal FMO value when added to the existing anchor beams will be selected as the next anchor point. Aiming to reduce the computational time we will only consider, at each iteration, the candidate beams that belong to the largest set of green beams between anchor points. The rationale of this idea is to add an anchor point where more degrees of freedom exist and, simultaneously, possibly reduce as much as possible the overall number of green points. For the nasopharyngeal tumor case used to illustrate our approach, the largest set of green beams is between the 4 th and the 5 th anchor point ( 54 candidate beams). By adding each of these green beams, one at a time, to the current set of anchor points we can compute the beam that leads to the best optimal FMO value and thus it is selected as new anchor point. In Fig. 4(a) the novel anchor point is displayed. This recently added red point leads to the infeasibility of some green beams due to the gantry/couch movement constraints here defined. At the end of each iteration, green candidate beams that became infeasible are removed as illustrated in Fig. 4(b). We are now in conditions to describe the algorithm for optimizing the noncoplanar arc trajectory. 


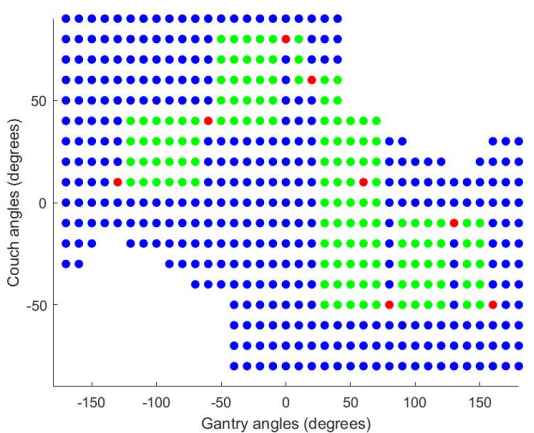

(a)

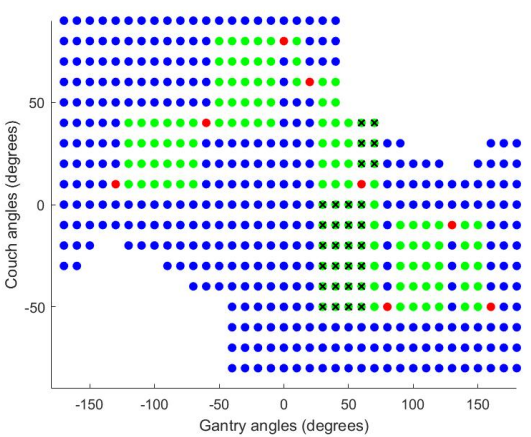

(b)

Fig. 4. Novel anchor point belonging to the largest set of green candidate beams is added $-4(\mathrm{a})$ and green candidate beams that became infeasible are removed $-4(\mathrm{~b})$.

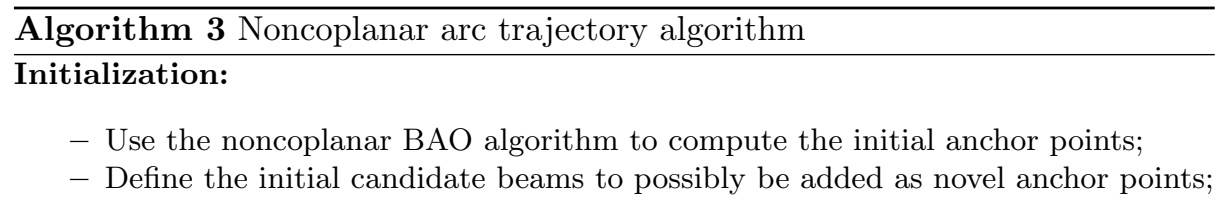

\section{Iteration:}

While candidate beams exist and number of anchor points is less than 20 do

1. Identify the largest set of candidate green beams between two anchor points;

2. Compute the optimal FMO value considering the set of beams composed of the anchor beams and each candidate beam identified in the previous step;

3. Add a novel anchor beam corresponding to the candidate beam that leads to the best optimal FMO value in the previous step;

4. Remove the green candidate beams that became infeasible.

\section{Computational Results}

Computational tests were conducted on a Dell Precision T5600 with Intel Xeon processor 64GB 1600MHz. An in-house MATLAB optimization suite, called YARTOS, developed at Erasmus MC Cancer Institute in Rotterdam, was used to compute dose distributions. YARTOS fluence optimizer, $2 p \epsilon c$, was used to calculate the optimal FMO value for a given set of beams. For the noncoplanar BAO problem, the initial step-size considered by the PSM algorithm was $\alpha_{0}=2^{5}=32$ while the minimal value allowed was one, leading to integer values of the beams.

For the nasopharyngeal tumor case used in our computational tests, three treatment plans were compared in this study: $4 \pi V M A T, 2 \pi V M A T$, and Equi, the typical seven-beam equispaced coplanar treatment plan used in step-and 


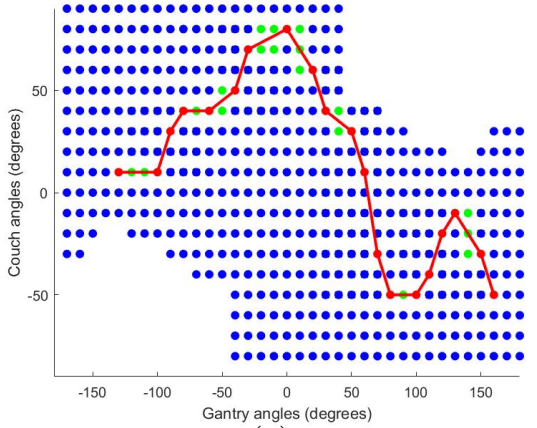

(a)

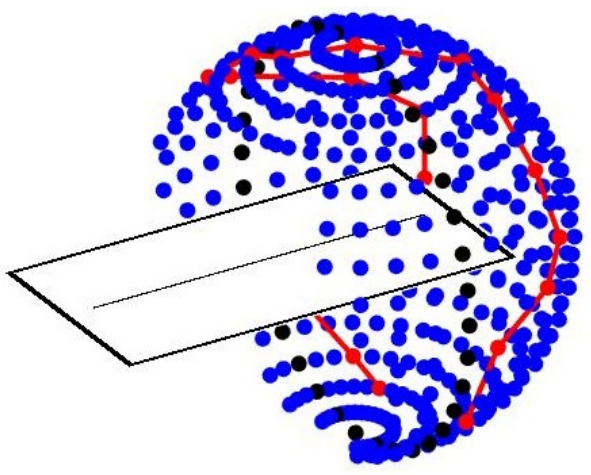

(b)

Fig. 5. Trajectory obtained by our noncoplanar arc trajectory optimization approach in $2 \mathrm{D}-5(\mathrm{a})$ and in $3 \mathrm{D}-5(\mathrm{~b})$.

Table 3. Results in terms of FMO objective function value.

\begin{tabular}{|c|c|c|c|c|}
\hline \multirow{2}{*}{$\frac{E q u i}{\text { FMO valu }}$} & \multicolumn{2}{|c|}{$2 \pi V M A T$} & \multicolumn{2}{|c|}{$4 \pi V M A T$} \\
\hline & MO val & lecre & MO val & decrease \\
\hline 560.33 & 522.19 & 6.8 & 473.92 & 15.4 \\
\hline
\end{tabular}

shoot IMRT. The final trajectory obtained by our noncoplanar arc trajectory optimization approach is displayed in Fig. 5.

Table 3 depicts the results, in terms of optimal FMO value, for the three treatment plans compared in this study. $4 \pi V M A T$ clearly outperforms the other treatment plans in terms of optimal FMO value, improving $15.4 \%$ the value obtained by Equi plan while the improvement of $2 \pi V M A T$ was $6.8 \%$.

The quality of the treatment plans is also acknowledged by a set of dose metrics. One of the metrics used for the tumors is coverage, the volume of PTV that receives $95 \%$ of the prescribed dose. At least $95 \%$ of the PTV volume is required. Other metrics typically screened for tumors are conformity and homogeneity that are output values of the YARTOS optimizer. Table 4 reports these tumor volume metrics. We can observe that slightly better target coverage, conformity and homogeneity numbers are obtained by $4 \pi V M A T$ treatment plan. For the OARs, depending on the type of organ, serial or parallel, maximum and/or mean doses are typically used to acknowledge organ sparing. Table 5 displays the organ sparing results. As expected the difference between plans is more visible for organ sparing. By simple inspection of Table 5 it is clear that $4 \pi$ VMAT treatment plan obtained by far the best organ sparing results. 
Table 4. Target coverage, conformity and homogeneity obtained by treatment plans.

\begin{tabular}{lccc}
\hline Target parameters & Equi & $2 \pi V M A T$ & $4 \pi V M A T$ \\
\hline$P T V_{70}$ Coverage & 0.863 & 0.849 & 0.919 \\
$P T V_{70}$ Conformity & 0.505 & 0.466 & 0.555 \\
$P T V_{70}$ Homogeneity & 0.880 & 0.873 & 0.892 \\
$P T V_{59.4}$ Coverage & 0.930 & 0.928 & 0.937 \\
$P T V_{59.4}$ Conformity & 0.554 & 0.551 & 0.562 \\
$P T V_{59.4}$ Homogeneity & 0.856 & 0.857 & 0.867 \\
\hline
\end{tabular}

Table 5. OARs sparing obtained by treatment plans.

\begin{tabular}{lcccccccc}
\hline & \multicolumn{3}{c}{ Mean Dose (Gy) } & & \multicolumn{3}{c}{ Max Dose (Gy) } \\
\cline { 2 - 4 } \cline { 7 - 8 } OAR & Equi & $2 \pi V M A T$ & $4 \pi V M A T$ & & Equi & $2 \pi V M A T$ & $4 \pi$ VMAT \\
\hline Spinal cord & - & - & - & & 34.9 & 33.6 & 30.8 \\
Brainstem & - & - & - & & 44.8 & 42.3 & 33.9 \\
Right parotid & 23.0 & 22.9 & 21.6 & & - & - & - \\
Left parotid & 24.4 & 19.3 & 15.4 & & - & - & - \\
Oral Cavity & 17.5 & 12.9 & 10.9 & & - & - & - \\
\hline
\end{tabular}

\section{Conclusions and Future Work}

A novel approach for the optimization of highly noncoplanar arc trajectories was described and tested using a complex nasopharyngeal tumor case already treated at IPOC. For the patient tested, the resulting noncoplanar arc plan, $4 \pi V M A T$, clearly outperforms both the coplanar arc plan, $2 \pi V M A T$, and the typically used coplanar equispaced step-and-shoot IMRT plan. Our approach gathers two extremely challenging problems: the noncoplanar BAO problem, a continuous global highly non-convex optimization problem, and the noncoplanar arc trajectory problem, a combinatorial problem yet to be solved satisfactorily. In this approach, we take advantage of all the quality work already produced for the noncoplanar BAO problem and propose an optimization strategy, anchored on the solution calculated by the BAO problem, that also considers dose metrics to guide the optimization procedure but simultaneously embeds the goal of obtaining an efficient dose delivery time, which is one of the main features of rotational treatments.

Although, for the patient at hand, the overall quality of the treatment is undoubtedly greater considering the noncoplanar arc plan, $4 \pi V M A T$, it comes with a cost. In terms of planning time, determining the optimal path of a highly noncoplanar arc plan following our optimization strategy is much more costly than simply using equispaced coplanar beams or using a coplanar arc (for a fixed couch angle of $0^{\circ}$ ). That was already the case for noncoplanar BAO in step-andshoot IMRT which is the main reason for the lack of BAO solutions in most of the treatment planning systems. Actually, angles are chosen manually, relying 
only on the experience of the planner, even in very challenging clinical cases. For this particular nasopharyngeal tumor case, the noncoplanar BAO procedure required the calculation of 2776 optimal FMO values (which consumes most of the computational time) while the optimization of the noncoplanar arc trajectory required the computation of 193 optimal FMO values. The number of function evaluations required in the second step of this two-step approach is $10 \%$ less than the number of function evaluations required for the first step, the noncoplanar BAO. Even knowing that the number of beams is increasing and thus the time required for each function evaluation also increases, in terms of computational time the second step is still $10 \%$ less than the noncoplanar BAO procedure (one hour against more than 10 hours). One way of speeding the overall optimization process is to consider less beams in the first step and that was precisely the reason for choosing 7 beams instead of 9 or more beams. In future work, the trade-off between the time required for noncoplanar BAO procedures - fastest if less beams (e.g., 5 beams) are considered or slowest BAO with more beams and the final quality of the corresponding treatment plans should be investigated. Moreover, other strategies to accelerate both the noncoplanar BAO procedure and the second step that determines the remaining anchor points should be investigated. Fully VMAT treatment plans should be compared as well in future

work. Finally, it is worth to note that this two-step optimization approach is completely automated which makes computational time somehow less important.

Acknowledgments. This work has been supported by project grant POCI-01-0145FEDER-028030 and by the Fundação para a Ciência e a Tecnologia (FCT) under project grant UID/Multi/00308/2019. The authors show gratitude to Ben Heijmen and Sebastiaan Breedveld for giving permission and helping them to install Erasmus-iCycle.

\section{References}

1. Bangert, M., Ziegenhein, P., Oelfke, U.: Comparison of beam angle selection strategies for intracranial imrt. Med. Phys. 40, 011716 (2013)

2. Bedford, J. L.: Treatment planning for volumetric modulated arc therapy, Med. Phys. 36, 5128-5138 (2009).

3. Otto, K.: Volumetric modulated arc therapy: IMRT in a single gantry arc, Med. Phys. 35, 310-317 (2008).

4. $\mathrm{Yu}, \mathrm{C}$. X.: Intensity-modulated arc therapy with dynamic multileaf collimation: An alternative to tomotherapy, Phys. Med. Biol. 40, 1435-1449 (1995)

5. Yang, Y., Zhang, P., Happersett, L., Xiong, J., Yang, J., Chan, M., Beal, K., Mageras, G., Hunt, M.: Choreographing couch and collimator in volumetric modulated arc therapy, Int. J. Radiat. Oncol. Biol. Phys. 80, 1238-1247 (2011)

6. MacDonald, R. L., Thomas, C. G.: Dynamic trajectory-based couch motion for improvement of radiation therapy trajectories in cranial SRT, Med. Phys. 42, $2317-2325$ (2015)

7. Smyth, G., Evans, P. M., Bamber, J. C., Mandeville, H. C., Welsh, L. C., Saran, F. H., Bedford, J. L.: Non-coplanar trajectories to improve organ at risk sparing 
in volumetric modulated arc therapy for primary brain tumors, Radiother. Oncol. 121, 124-131 (2016)

8. Smyth, G., Bamber, J. C., Evans, P. M., Bedford, J. L.: Trajectory optimisation for dynamic couch rotation during volumetric modulated arc radiotherapy, Phys. Med. Biol. 58, 8163-8177 (2013)

9. Papp, D., Bortfeld, T., Unkelbach, J.: A modular approach to intensity-modulated arc therapy optimization with noncoplanar trajectories, Phys. Med. Biol. 60, 51795198 (2015)

10. Wild, E., Bangert, M., Nill, S., Oelfke, U.: Noncoplanar VMAT for nasopharyngeal tumors: Plan quality versus treatment time. Med. Phys. 42, 2157-2168 (2015)

11. Langhans, M., Unkelbach, J., Bortfeld, T., Craft, D.: Optimizing highly noncoplanar VMAT trajectories: the NoVo method, Phys. Med. Biol. 63025023 (2018)

12. Rocha, H., Dias, J., Ventura, T., Ferreira, B.C., Lopes, M.C.: A derivative-free multistart framework for an automated noncoplanar beam angle optimization in IMRT. Med. Phys. 43, 5514-5526 (2016)

13. Dias, J., Rocha, H., Ventura, T., Ferreira, B.C., Lopes, M.C.: Automated fluence map optimization based on fuzzy inference systems. Med. Phys. 43, 1083-1095 (2016)

14. Breedveld, S., Storchi, P., Keijzer, M., Heemink, A.W., Heijmen, B.: A novel approach to multi-criteria inverse planning for IMRT. Phys. Med. Biol. 52, 6339-6353 (2007)

15. Breedveld, S., Storchi, P., Heijmen, B.: The equivalence of multicriteria methods for radiotherapy plan optimization. Phys. Med. Biol. 54, 7199-7209 (2009)

16. Breedveld, S., Storchi, P., Voet, P., Heijmen, B.: iCycle: integrated, multicriterial beam angle, and profile optimization for generation of coplanar and noncoplanar IMRT plans. Med. Phys. 39, 951-963 (2012)

17. Dias, J., Rocha, H., Ferreira, B.C., Lopes, M.C.: Simulated annealing applied to IMRT beam angle optimization: A computational study. Phys. Med. 31, 747-756 (2015)

18. Aleman, D.M., Kumar, A., Ahuja, R.K., Romeijn, H.E., Dempsey, J.F.: Neighborhood search approaches to beam orientation optimization in intensity modulated radiation therapy treatment planning. J. Global Optim. 42, 587-607 (2008)

19. Craft, D.: Local beam angle optimization with linear programming and gradient search. Phys. Med. Biol. 52, 127-135 (2007)

20. Lim, G.J., Cao, W.: A two-phase method for selecting IMRT treatment beam angles: Branch-and-Prune and local neighborhood search. Eur. J. Oper. Res. 217, 609-618 (2012)

21. Dias, J., Rocha, H., Ferreira, B.C., Lopes, M.C.: A genetic algorithm with neural network fitness function evaluation for IMRT beam angle optimization. Cent. Eur. J. Oper. Res. 22, 431-455 (2014)

22. Bertsimas, D., Cacchiani, V., Craft, D., Nohadani, O.: A hybrid approach to beam angle optimization in intensity-modulated radiation therapy. Comput. Oper. Res. 40, 2187-2197 (2013)

23. Bangert, M., Ziegenhein, P., Oelfke, U.: Characterizing the combinatorial beam angle selection problem. Phys. Med. Biol. 57, 6707-6723 (2012)

24. Rocha, H., Dias, J., Ferreira, B.C., Lopes, M.C.: Selection of intensity modulated radiation therapy treatment beam directions using radial basis functions within a pattern search methods framework. J. Glob. Optim. 57, 1065-1089 (2013)

25. Rocha, H., Dias, J., Ferreira, B.C., Lopes, M.C.: Beam angle optimization for intensity-modulated radiation therapy using a guided pattern search method. Phys. Med. Biol. 58, 2939-2953 (2013) 
26. Rocha, H., Dias, J., Ferreira, B.C., Lopes, M.C.: Pattern search methods framework for beam angle optimization in radiotherapy design. Appl. Math. Comput. 219, 10853-10865 (2013)

27. Rocha, H., Dias, J., Ferreira, B.C., Lopes, M.C.: Noncoplanar beam angle optimization in IMRT treatment planning using pattern search methods, J. Phys.: Conf. Ser. 616, 012014 (2015)

28. Rocha, H., Dias, J., Ventura, T., Ferreira, B.C., Lopes, M.C.: Beam angle optimization in IMRT: are we really optimizing what matters?, Int. Trans. Oper. Res. 26, 908-928 (2019)

29. Rocha, H., Dias, J., Ventura, T., Ferreira, B.C., Lopes, M.C.: Comparison of combinatorial and continuous frameworks for the beam angle optimization problem in IMRT. In: Gervasi, O., et al. (eds.) ICCSA 2018. LNCS, vol. 10961, pp. 593606. Springer, Cham (2018) 